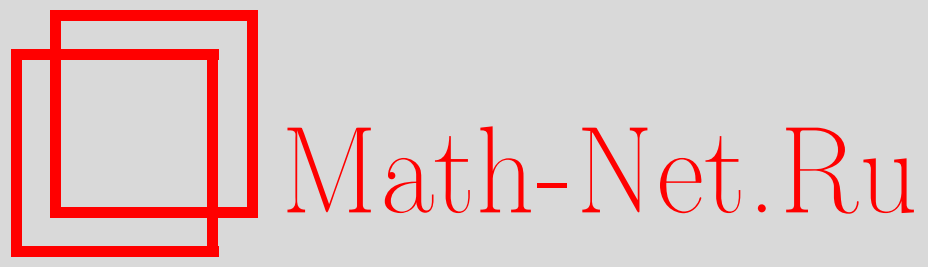

Г. Ю. Софронов, Асимптотически $d$-оптимальный критерий в задаче апостериорного обнаружения разладки, Теория вероятн. и ее примен., 2004, том 49, выпуск 2, 396-399

DOI: https://doi.org/10.4213/tvp229

Использование Общероссийского математического портала Math-Net.Ru подразумевает, что вы прочитали и согласны с пользовательским соглашением

http://www . mathnet.ru/rus/agreement

Параметры загрузки:

IP: 52.90 .164 .192

26 апреля 2023 г., 14:14:45

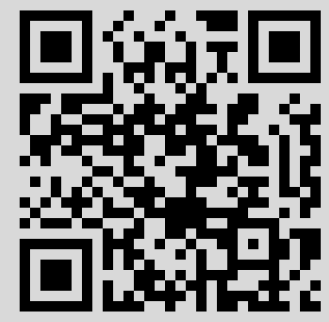


(C) $2004 \mathrm{r}$.

СОФРОНОВ Г. Ю."

\section{АСИМПТОТИЧЕСКИ $d$-ОПТИМАЛЬНЫЙ КРИТЕРИЙ В ЗАДАЧЕ АПОСТЕРИОРНОГО ОБНАРУЖЕНИЯ РАЗЛАДКИ}

Рассматривается задача апостериорного обнаружения разладки для последовательности независимых случайных величин. Вместо ошибок первого и второго рода предлагается использовать $d$-риски. Строится асимптотически оптимальный критерий, минимизируюший один $d$-риск и гарантирующий другой.

Ключевые слова и фразы: обнаружение разладки, различение гипотез, сближаюшиеся гипотезы, $d$-апостериорный подход, $d$-оптимальность, слабая сходимость, функционалы от винеровского процесса.

1. Постановка задачи. Пусть задано вероятностное пространство $\left(\Omega, \mathscr{F}, \mathbf{P}_{\theta}\right)$, $\theta$ принимает значения в открытом подмножестве $\Theta$ евклидова пространства $\mathbf{R}^{1}$, $X^{(n)}=\left(X_{1}, \ldots, X_{n}\right)-$ случайный вектор с независимыми компонентами.

Рассматривается задача (см., например, обзорную статью [1]) проверки гипотезы $H_{0}$ (отсутствие разладки): независимые случайные величины $X_{1}, \ldots, X_{n}$ одинаково распределены с функцией распределения $F_{\theta_{0}}(x)$, при альтернативе $H_{1}$ (есть разлацка): $X_{1}, \ldots, X_{\tau}$ распределены с функцией распределения $F_{\theta_{0}}(x)$, a $X_{\tau+1}, \ldots, X_{n}-$ с функцией распределения $F_{\theta_{1}}(x)$, где $\theta_{0} \neq \theta_{1}$. В дальнейшем будем считать, что значение параметра до разладки $\theta=\theta_{0}$ известно. В [2] рассматривался случай, когда $\theta$ - параметр сдвига. Предполагается, что момент разладки $\tau$ есть случайная величина с некоторым априори известным распределением вероятностей $\mathbf{P}\{\tau=k\}=p_{k}$, $k=0,1, \ldots, n$, которое удовлетворяет следуюшему условию: const.

(A) $\lim _{n \rightarrow \infty} n p_{k}=p(t)$ при $k / n \rightarrow t, 0 \leqslant t<1, k=0,1, \ldots, n-1, \lim _{n \rightarrow \infty} p_{n}=p \equiv$

Например, это требование выполняется для часто используемого в задачах о разладке геометрического распределения $p_{k}=q(1-q)^{k}, k=0,1, \ldots, n-1, p_{n}=(1-q)^{n}$, где $q$ - вероятность разладки на каждом шаге. В этом случае при $n q=\lambda$

$$
\lim _{n \rightarrow \infty} n p_{k}=p(t)=\lambda e^{-\lambda t}, \quad k=0,1, \ldots, n-1, \quad \lim _{n \rightarrow \infty} p_{n}=p=e^{-\lambda} .
$$

Событие $\{\tau=n\}$ означает, что разладка не произошла (справедлива гипотеза $H_{0}$ ), $p_{n}$ - вероятность отсутствия разладки.

Будем считать, что функция распределения $F_{\theta}(x)$ имеет плотность $f_{\theta}(x)$ (по некоторой $\sigma$-конечной мере $\mu$ ). Выполнены следующие стандартные условия регулярности.

$\left(\mathrm{B}_{1}\right)$ Функция плотности $f_{\theta}(x) \mu$-п.в. непрерывна при любом $\theta \in \Theta$.

$\left(\mathrm{B}_{2}\right)$ Функиия $\sqrt{f_{\theta}(x)}$ непрерывно дифференцируема в. каждой точке $\theta \in \Theta$ ө смьсле $L_{2}$ (имеет конечную информачию Фишера $I(\theta)$ при любом $\theta \in \Theta$ ).

$\left(\mathrm{B}_{3}\right)$ Функиия $f_{\theta}(x)$ дважды непрерывно дифференцируема по параметру под знаком интеграла.

$\left(\mathrm{B}_{4}\right)$ Интеграл $I(\theta)=\int\left(f_{\theta}^{\prime}(x) / f_{\theta}(x)\right)^{2} f_{\theta}(x) d \mu(x)$ равномерно сходится.

При различении гипотез $H_{0}$ и $H_{1}$ вместо традиционных ошибок первого и второго рода предлагается использовать $d$-риски (см. подробнее обзор [3]):

$$
R_{0}=\mathbf{P}\left\{\tau=n \mid \text { принята } H_{1}\right\}, \quad R_{1}=\mathbf{P}\left\{\tau<n \mid \text { принята } H_{0}\right\} .
$$

* Марийский государственный университет, пл. Ленина, 1, 424001 Йошкар-Ола, Марий Эл, РФ; e-mail: gsofronov@marsu.ru

1) Работа выполнена при поддержке гранта НШ-1758.2003.1 Программы поддержки ведуших научных школ России. 
Как было показано в [4], оптимальный критерий $\varphi_{n}\left(X^{(n)}\right)$, гарантирующий $d$ риск $R_{1} \leqslant \beta$ и минимизирующий $d$-риск $R_{0}$, основан на статистике $T_{n}=T_{n}\left(X^{(n)}\right)=$ $\mathbf{P}\left\{\tau=n \mid X^{(n)}\right\}$ и имеет вид

$$
\varphi_{n}=\varphi_{n}\left(X^{(n)}\right)=\left\{\begin{array}{lll}
1, & \text { если } & T_{n}<c_{n}, \\
\gamma_{n}, & \text { если } & T_{n}=c_{n}, \\
0, & \text { если } T_{n}>c_{n} .
\end{array}\right.
$$

Будем искать асимптотику критерия $\varphi_{n}$ при сближающихся гипотезах $H_{0}$ и $H_{1}$. В связи с этим рассмотрим два возможных варианта. Основной результат относится к случаю, когда $\theta_{1}=\theta_{0}+\delta / \sqrt{n}$, где $\delta$ - постоянная. В случае с дополнительной байесовостью будем считать, что значение параметра $\theta_{1}$ является реализацией случайной величины с плотностью $g(\theta)$. Поэтому близость гипотез естественно связать со степенью сосредоточенности априорного распределения в точке $\theta_{0}$. Для этого предлагается использовать (см. [5]) распределение вида $g(\theta)=\sigma^{-1} g\left(\left(\theta-\theta_{0}\right) / \sigma ; \sigma\right)$, где $\sigma>0$ - параметр, характеризующий степень концентрации априорного распределения в точке $\theta_{0}$.

2. Основной результат. Предположим, что $\theta_{1}=\theta_{0}+\delta / \sqrt{n}$, где $\delta$ - постоянная. Введем обозначения:

$$
H_{n}\left(c_{n} \mid \tau=k_{0}\right)=\mathbf{P}\left\{T_{n}<c_{n} \mid \tau=k_{0}\right\}, \quad k_{0}=0,1, \ldots, n,
$$

-- функция распределения статистики $T_{n}$,

$L_{n}\left(c_{n} \mid \tau=k_{0}\right)=1-H_{n}\left(c_{n} \mid \tau=k_{0}\right)+\left(1-\gamma_{n}\right)\left(H_{n}\left(c_{n}+0 \mid \tau=k_{0}\right)-H_{n}\left(c_{n} \mid \tau=k_{0}\right)\right)$

- оперативная характеристика критерия $\varphi_{n}$. Тогда несложно получить, что

$$
\begin{aligned}
& R_{0}\left(n, c_{n}, \gamma_{n}\right)=\mathbf{P}\left\{\tau=n \mid \varphi_{n}=1\right\}=\frac{p_{n}\left(1-L_{n}\left(c_{n} \mid \tau=n\right)\right)}{\sum_{k_{0}=0}^{n} p_{k_{0}}\left(1-L_{n}\left(c_{n} \mid \tau=k_{0}\right)\right)}, \\
& R_{1}\left(n, c_{n}, \gamma_{n}\right)=\mathbf{P}\left\{\tau<n \mid \varphi_{n}=0\right\}=\frac{\sum_{k_{0}=0}^{n-1} p_{k_{0}} L_{n}\left(c_{n} \mid \tau=k_{0}\right)}{\sum_{k_{0}=0}^{n} p_{k_{0}} L_{n}\left(c_{n} \mid \tau=k_{0}\right)} .
\end{aligned}
$$

Константы $c_{n}$ и $\gamma_{n}$ находятся из равенства $R_{1}\left(n, c_{n}, \gamma_{n}\right)=\beta$. В [6] показывается, что $\left\{c_{n}\right\}$ - сходящаяся к некоторому $c$ последовательность. Введем обозначения: $\tau^{\prime}=\tau / n-$ случайная величина, соответствующая $\tau, \tau \in\{0,1 \ldots, n\}, H\left(c \mid \tau^{\prime}=t_{0}\right)=$ $\lim _{n \rightarrow \infty} H_{n}\left(c_{n} \mid \tau=k_{0}\right), L\left(c \mid \tau^{\prime}=t_{0}\right)=\lim _{n \rightarrow \infty} L_{n}\left(c_{n} \mid \tau=k_{0}\right)$ при $k_{0} / n \rightarrow t_{0}$. Основную роль при дальнейших рассуждениях будут играть случайные процессы

$$
\begin{aligned}
Z_{1}(u, t) & =\exp \left(u \sqrt{I\left(\theta_{0}\right)} W(1-t)-\frac{u^{2}}{2} I\left(\theta_{0}\right)(1-t)\right), \\
Z_{t_{0}}(u, t) & =\exp \left(u \sqrt{I\left(\theta_{0}\right)} W(1-t)+\frac{u^{2}}{2} I\left(\theta_{0}\right) h\left(t_{0}, t\right)\right), \\
h\left(t_{0}, t\right) & = \begin{cases}1+t-2 t_{0}, & t<t_{0}, \\
1-t, & t \geqslant t_{0},\end{cases}
\end{aligned}
$$

где $I\left(\theta_{0}\right)$ - информация Фишера в точке $\theta_{0}, W(\cdot)$ - стандартный винеровский процесc.

Нетрудно доказать следующую лемму (см. также [7, теорема 11]).

Лемма 1. При выполнении условий $\left(\mathrm{B}_{1}\right)-\left(\mathrm{B}_{4}\right) u \theta_{1}=\theta_{0}+\delta / \sqrt{n}$, где $\delta-$ nостоянная, имеет место:

1) слабая сходимость по распределению $\mathbf{P}_{\theta_{0}}$ :

$$
\exp \left(\sum_{i=[n t]+1}^{n} \ln \frac{f_{\theta_{1}}\left(X_{i}\right)}{f_{\theta_{0}}\left(X_{i}\right)}\right) \stackrel{\mathrm{d}}{\longrightarrow} Z_{1}(\delta, t),
$$

2) слабая сходимость по распределению $\mathbf{P}_{\theta_{1}}$ :

$$
\exp \left(\sum_{i=[n t]+1}^{n} \ln \frac{f_{\theta_{1}}\left(X_{i}\right)}{f_{\theta_{0}}\left(X_{i}\right)}\right) \stackrel{\mathrm{d}}{\longrightarrow} Z_{t_{0}}(\delta, t) .
$$


Теорема 1. Пусть выполнены условие (A) и условия леммы 1. Если $c_{n} \rightarrow c$ при $n \rightarrow \infty$, mo

$$
\begin{aligned}
\lim _{n \rightarrow \infty} R_{0}\left(n, c_{n}, \gamma_{n}\right) & =R_{0}(c)=\frac{p\left(1-L\left(c \mid \tau^{\prime}=t_{0}\right)\right)}{\int_{0}^{1} p\left(t_{0}\right)\left(1-L\left(c \mid \tau^{\prime}=t_{0}\right)\right) d t_{0}}, \\
\lim _{n \rightarrow \infty} R_{1}\left(n, c_{n}, \gamma_{n}\right) & =R_{1}(c)=\frac{\lim _{a \rightarrow 1-0} \int_{0}^{a} p\left(t_{0}\right) L\left(c \mid \tau^{\prime}=t_{0}\right) d t_{0}}{\int_{0}^{1} p\left(t_{0}\right) L\left(c \mid \tau^{\prime}=t_{0}\right) d t_{0}}, \\
H\left(c \mid \tau^{\prime}=t_{0}\right) & =\mathbf{P}\left\{T<c \mid \tau^{\prime}=t_{0}\right\}=\mathbf{P}\left\{\int_{0}^{1} p(t) Z_{t_{0}}(\delta, t) d t>\frac{p}{c}\right\},
\end{aligned}
$$

где постоянная с выбирается ках решение уравнения $R_{1}(c)=\beta$.

Д о к а з а т е л с с в о. Применяя формулу Байеса, найдем апостериорную вероятность отсутствия разладки:

$$
\begin{aligned}
\mathbf{P}\left\{\tau=n \mid X^{(n)}\right\} & =\frac{p_{n} \prod_{i=1}^{n} f_{\theta_{0}}\left(X_{i}\right)}{\sum_{k=0}^{n} p_{k} \prod_{i=1}^{k} f_{\theta_{0}}\left(X_{i}\right) \prod_{i=k+1}^{n} f_{\theta_{1}}\left(X_{i}\right)} \\
& =\frac{1}{p_{n}^{-1} \sum_{k=0}^{n} p_{k} \exp \left(\sum_{i=k+1}^{n} \ln \left(f_{\theta_{1}}\left(X_{i}\right) / f_{\theta_{0}}\left(X_{i}\right)\right)\right)} .
\end{aligned}
$$

Далее, условие (A) и лемма 1 вместе с теоремой 1 работы [8] дают

$\lim _{n \rightarrow \infty} \mathbf{P}\left\{p_{n}^{-1} \sum_{k=0}^{n} \frac{1}{n} n p_{k} \exp \left(\sum_{i=k+1}^{n} \ln \frac{f_{\theta_{1}}\left(X_{i}\right)}{f_{\theta_{0}}\left(X_{i}\right)}\right)<x\right\}=\mathbf{P}\left\{p^{-1} \int_{0}^{1} p(t) Z_{t_{0}}(\delta, t) d t<x\right\}$.

Тогда $T_{n}\left(X^{(n)}\right)$ слабо сходится к случайной величине $T$ с распределением

$$
H\left(c \mid \tau^{\prime}=t_{0}\right)=\mathbf{P}\left\{T<c \mid \tau^{\prime}=t_{0}\right\}=\mathbf{P}\left\{\int_{0}^{1} p(t) Z_{t_{0}}(\delta, t) d t>\frac{p}{c}\right\} .
$$

Таким образом, постоянная рандомизации $\gamma$ не влияет на приведенную асимптотику.

Полученные результаты позволяют представить асимптотики $d$-рисков в виде

$$
\begin{aligned}
& R_{0}(c)=\lim _{n \rightarrow \infty} R_{0}\left(n, c_{n}, \gamma_{n}\right)=\frac{p\left(1-L\left(c \mid \tau^{\prime}=t_{0}\right)\right)}{\int_{0}^{1} p\left(t_{0}\right)\left(1-L\left(c \mid \tau^{\prime}=t_{0}\right)\right) d t_{0}}, \\
& R_{1}(c)=\lim _{n \rightarrow \infty} R_{1}\left(n, c_{n}, \gamma_{n}\right)=\frac{\lim _{a \rightarrow 1-0} \int_{0}^{a} p\left(t_{0}\right) L\left(c \mid \tau^{\prime}=t_{0}\right) d t_{0}}{\int_{0}^{1} p\left(t_{0}\right) L\left(c \mid \tau^{\prime}=t_{0}\right) d t_{0}},
\end{aligned}
$$

где постоянную $c$ следует выбирать как решение уравнения $R_{1}(c)=\beta$. В [6] доказано, что существует единственное решение этого уравнения. Теорема доказана.

3. Случай с дополнительной байесовостью. Будем считать, что значение параметра $\theta_{1}$ является реализацией случайной величины с плотностью $g(\theta)=$ $\sigma^{-1} g\left(\left(\theta-\theta_{0}\right) / \sigma ; \sigma\right)$, где $\sigma>0$ - параметр, характеризуюший степень концентрации априорного распределения в точке $\theta_{0}$. Кроме того, выполнено следуюшее условие регулярности.

(C) Семейство плотностей $\{g(\cdot, \sigma), \sigma>0\}$ имеет предельную плотность $g(u, 0)=\lim _{\sigma \rightarrow 0} g(u, \sigma)$.

В [5] показано, что этому требованию удовлетворяет, например, бетараспределение

$$
g(\theta)=\frac{1}{\sigma} g\left(\frac{\theta-\theta_{0}}{\sigma} ; \sigma\right)=\frac{1}{B\left(\lambda_{1}, \lambda_{2}\right)} \theta^{\lambda_{1}-1}(1-\theta)^{\lambda_{2}-1}, \quad 0<\theta<1,
$$

причем $\lambda_{1}$ и $\lambda_{2}$ таковы, что $\mathbf{E} \theta_{1}=\theta_{0}=\lambda_{1} /\left(\lambda_{1}+\lambda_{2}\right)$. Если определить $\sigma^{2}$ равным $\mathbf{D} \theta_{1}=\theta_{0}\left(1-\theta_{0}\right) /\left(1+\lambda_{1}+\lambda_{2}\right)$, то при $\sigma \rightarrow 0$ значения $\lambda_{1}$ и $\lambda_{2}$ стремятся к бесконечности так, что $g(u, 0)=(2 \pi)^{-1 / 2} \exp \left(-u^{2} / 2\right)$.

Следуюшая лемма доказывается по аналогии с леммой 1 из [5]. 
Лемма 2. Пусть $r_{n}-$ последовательность неотричательньх чисел, сходящаяся $x$ некоторому $r>0, u \theta_{1}=\theta_{0}+u \sigma_{n}, \sigma_{n}=r_{n} / \sqrt{n}$. При выполнении условиц $\left(\mathrm{B}_{1}\right)-\left(\mathrm{B}_{4}\right),(\mathrm{C})$ uмеет место:

1) слабая сходимость по распределению $\mathbf{P}_{\theta_{0}}$ :

$$
\int_{-\infty}^{\infty} \exp \left(\sum_{i=[n t]+1}^{n} \ln \frac{f_{\theta_{0}+u \sigma_{n}}\left(X_{i}\right)}{f_{\theta_{0}}\left(X_{i}\right)}\right) g\left(u ; \sigma_{n}\right) d u \stackrel{\mathrm{d}}{\longrightarrow} \int_{-\infty}^{\infty} Z_{1}(u r, t) g(u ; 0) d u,
$$

2) слабая сходимость по распределению $\mathbf{P}_{\theta_{1}}$ :

$$
\int_{-\infty}^{\infty} \exp \left(\sum_{i=[n t]+1}^{n} \ln \frac{f_{\theta_{0}+u \sigma_{n}}\left(X_{i}\right)}{f_{\theta_{0}}\left(X_{i}\right)}\right) g\left(u ; \sigma_{n}\right) d u \stackrel{\mathrm{d}}{\longrightarrow} \int_{-\infty}^{\infty} Z_{t_{0}}(u r, t) g(u ; 0) d u .
$$

В заключение сформулируем утверждение, которое является аналогом теоремы 1.

Теорема 2. Пусть выполнены условие (A) и условия леммы 2. Если $c_{n} \rightarrow c$ npu $n \rightarrow \infty$, mo

$$
\begin{aligned}
H\left(c \mid \tau^{\prime}=t_{0}\right) & =\mathbf{P}\left\{\frac{\int_{-\infty}^{\infty} g(u ; 0) d u}{p^{-1} \int_{0}^{1} p(t) \int_{-\infty}^{\infty} Z_{t_{0}}(u r, t) g(u ; 0) d u d t}<c\right\} \\
& =\mathbf{P}\left\{\int_{0}^{1} p(t) \int_{-\infty}^{\infty} Z_{t_{0}}(u r, t) g(u ; 0) d u d t>\frac{p}{c}\right\}
\end{aligned}
$$

постоянная с выбирается как решение уравнения $R_{1}(c)=\beta$.

Следствие. Если

mo

$$
g(u, 0)=(2 \pi)^{-1 / 2} \exp \left(-\frac{u^{2}}{2}\right)
$$

$$
\begin{aligned}
& H\left(c \mid \tau^{\prime}=t_{0}\right)=\mathbf{P}\left\{T<c \mid \tau^{\prime}=t_{0}\right\} \\
& \quad=\mathbf{P}\left\{\int_{0}^{1} \frac{p(t)}{\sqrt{1-r^{2} I\left(\theta_{0}\right) h\left(t_{0}, t\right)}} \exp \left(\frac{r^{2} I\left(\theta_{0}\right) W^{2}(1-t)}{2\left(1-r^{2} I\left(\theta_{0}\right) h\left(t_{0}, t\right)\right)}\right) d t>\frac{p}{c}\right\} .
\end{aligned}
$$

\section{СПИСОК ЛИТЕРАТУРЫ}

1. Колмогоров А.Н., Прохоров Ю.В., Ширяев А.Н. Вероятностно-статистические методы обнаружения спонтанно возникаюших эффектов. - Труды МИАН, 1988, T. 182, c. $4-23$.

2. Софронов Г.Ю. Асимптотически $d$-оптимальный критерий обнаружения разладки. - Теория вероятн. и ее примен., 2001, т. 46, в. 3, с. 571-572.

3. Володин И. Н., Новиков А. A., Симушкин C. В. Гарантийный статистический контроль качества: апостериорный подход. - Обозрение прикл. и промышл. матем., сер. вероятн. и статист., 1994, т. 1, в. 2, с. 148-178.

4. Симушкин C. В. Эмпирический $d$-апостериорный подход к проблеме гарантийности статистического вывода. - Изв. вузов, сер. матем., 1983, № 11, с. 42-58.

5. Володин И.Н., Новиков А.А. Асимптотика необходимого объема выборки при $d$-гарантийном различении двух гипотез. - Изв. вузов, сер. матем., 1983, № 11, c. $59-66$.

6. Володин И.Н., Новиков Ан. А. Асимптотика необходимого объема выборки при гарантийном различении параметрических гипотез. - Исследования по прикладной математике. Казань: Изд-во Казанского ун-та, 1999, в. 21, с. 3-41.

7. Greenwood P.E., Shiryayev A.N. Contiguity and the Statistical Invariance Principle. New York: Gordon \& Breach, 1985, 236 p.

8. MacNeill I. B. Tests for change of parameter at unknown times and distributions of some related functionals on Brownian motion. - Ann. Statist., 1974, v. 2, № 5, p. $950^{\circ}-962$. 